\title{
Embedded progressive-three-layered fiber long-period gratings for respiratory monitoring
}

\author{
Thomas Allsop \\ T. Earthrowl-Gould \\ David J. Webb \\ Ian Bennion \\ Aston University \\ Photonics Research Group \\ Aston Triangle \\ Birmingham B4 7ET \\ United Kingdom \\ E-mail: t.d.p.allsop@aston.ac.uk
}

\begin{abstract}
A long-period grating (LPG) was written into a progressive three-layered single-mode fiber that was embedded into a flexible platform as a curvature sensor. The spectral location and profile of the LPGs were unaltered after implantation in the platform. The curvature sensitivity was $3.747 \mathrm{~nm} \mathrm{~m}$ with a resolution of $\pm 1.1 \times 10^{-2} \mathrm{~m}^{-1}$. The bend sensor is intended to be part of a respiratory monitoring system and was tested on a resuscitation training manikin. () 2003 Society of Photo-Optical Instrumentation Engineers. [DOI: 10.1117/1.1578497]

Keywords: curvature sensing, long-period gratings, respiratory monitoring.

Paper JBO 02053 received Aug. 7, 2002; revised manuscript received Jan. 7, 2003; accepted for publication Jan. 31, 2003.
\end{abstract}

\section{Introduction}

A long-period grating (LPG) is a UV light induced periodic modulation of the refractive index of the core of a singlemode optical fiber with a modulation depth of about $10^{-4}$ and a period typically between 100 and $600 \times 10^{-6} \mathrm{~m}$. The effect of the LPG is to introduce a number of attenuation bands into the fiber's transmissivity, occurring at wavelengths where the LPG enables phase-matched coupling between the guided core mode and cladding modes. ${ }^{1}$ The study of the LPG's attenuation bands has yielded many potential applications. One important area is in fiber-optic telecommunications, where, for example, they have found use as wavelength-selective filters for flattening the gain spectrum of an erbium-doped fiber amplifier. $^{2}$ Over the past few years, the LPG has also found numerous applications in the field of sensing through its sensitivities to strain, bending and shape, temperature, and the refractive index of the surrounding medium. ${ }^{3-7}$ The LPG sensitivity can manifest itself in two ways; first through the spectral shift in the attenuation band, which we refer to here as spectral sensitivity, and second through a change in the strength of the attenuation band, which we refer to as a spectral profile variation.

In general, problems can arise when using an LPG that is written into a conventional step-index single-mode fiber, owing to its spectral sensitivity to external influences. For telecommunications and structural monitoring applications, problems occur when the fiber is recoated after the inscription process or embedded in another material. In a step-index fiber, the spectral position and strength of the LPG attenuation bands are dependent upon the surrounding medium's refractive index $\left(n_{s}\right)$ and in the process of recoating the fiber, $n_{s}$ changes, thereby altering the spectral profile and position of the attenuation bands. In some cases the attenuation bands may even disappear. To overcome such problems, there has been some interest in the spectral behavior of LPGs fabricated in multilayered single mode optical fibers. Examples include the use of air-clad ${ }^{8}$ or depressed-ladding ${ }^{9}$ fibers to desensitize the structure to changes in the refractive index of the surrounding medium.

Recent reports on the fabrication of LPGs in a single-mode progressive three-layered (PTL) fiber have shown that the lower order cladding modes are insensitive to $n_{s} .{ }^{10}$ In this paper, for the first time to the authors' knowledge, experimental data relating to temperature and bending are presented for an embedded LPG written in the PTL fiber. The intended application for this device is as a shape sensor in monitoring respiratory function, where the movement of the torso during respiration must be monitored. The measurement of lung function is generally based upon the use of spirometric devices that either measure the volume of gases expired (or inspired) at the mouth, or the corresponding flow rate. The measured output requires that an individual blow forcibly into a mouthpiece attached to a recording system and as such provides no more than an averaged representation of lung function behavior and the condition of the respiratory system as a whole. ${ }^{11-12}$ It is desirable to obtain detailed information on the contribution to breathing of different regions of the chest and abdomen, ${ }^{12}$ and spirometric devices do not provide this information.

The sensor investigated in this work is intended to be a component of a multiplexed system capable of assessing lung function that avoids the need for flow measurement at the mouth and provides more detailed information on the dynamics of chest motion during breathing. Such a system would facilitate further studies of respiratory physiology since no product is currently available that can provide a completely noninvasive and quantitative appreciation of respiratory function. $^{12}$

\section{Fabrication and Transmission Spectrum of the LPG Sensor}

The PTL optical fiber was supplied by Fibercore Ltd. and was originally designed for a cladding-pumped, erbium-doped fiber laser. This fiber has a core of $\mathrm{GeO}_{2} / \mathrm{SiO}_{2}$ with a radius of $2.6 \times 10^{-6} \mathrm{~m}$, an inner cladding of $\mathrm{SiO}_{2}$ with a radius of $45.2 \times 10^{-6} \mathrm{~m}$, and an outer cladding of $\mathrm{SiO}_{2} / \mathrm{F} / \mathrm{P}_{2} \mathrm{O}_{5}$ with a radius of $62.5 \times 10^{-6} \mathrm{~m}$. The fiber is not specifically designed to be photosensitive and so its photosensitivity was increased by hydrogenation at a pressure of 120 bar for a period of two weeks at room temperature. A number of LPGs of various

1083-3668/2003/\$15.00 @ 2003 SPIE 


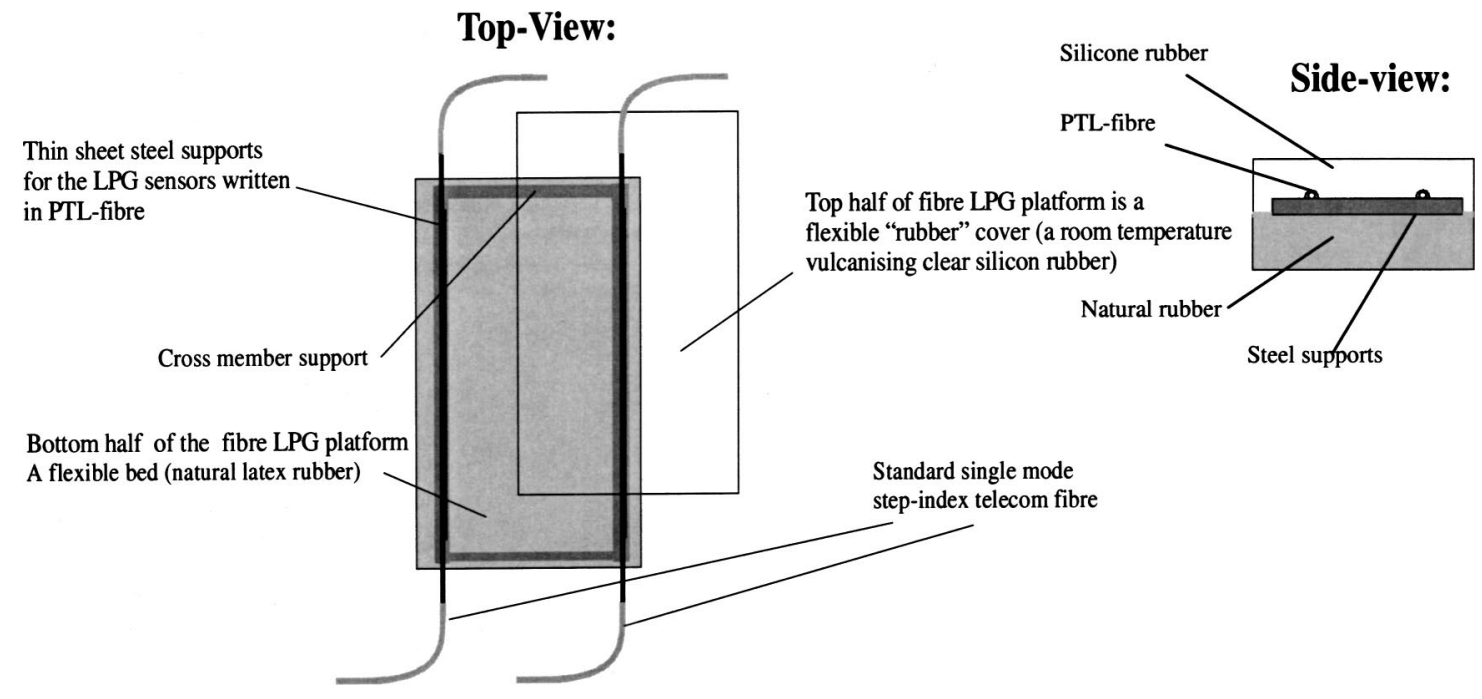

Fig. 1 The construction of the long-period grating sensor.

lengths and periods were fabricated in the fiber using the point-by-point writing technique ${ }^{13}$; a programmable shutter provided this facility. The UV writing beam was generated from a frequency-doubled argon ion laser and its power of 70 $\mathrm{mW}$ was focused to a size of approximately $10 \times 10^{-6} \mathrm{~m}$.

The periods of the LPGs were chosen so that the attenuation bands associated with cladding modes $n_{c l}(1,1)$ to $n_{c l}(1,10)$, which are insensitive to $n_{s},{ }^{14}$ were spectrally located in the range 1400-1550 $\mathrm{nm}$ to facilitate interrogation. Two LPGs with periods of $350 \times 10^{-6} \mathrm{~m}$ and $240 \times 10^{-6} \mathrm{~m}$ were embedded to produce a sensor.

Details of the sensor construction are shown in Fig. 1. Assembly was carried out on a base pad of natural latex rubber 250 by $120 \mathrm{~mm}$ and with a $2-\mathrm{mm}$ depth. This provided both a flexible stage as well as a thermally insulating layer for monitoring respiratory function (reducing the effects of rapid ambient temperature fluctuations). The two LPGs were fixed using a standard cyanoacrylate adhesive to strips of carbon steel $200 \times 12 \times 0.254 \mathrm{~mm}$. The LPGs were placed in straight grooves along the length of the steel strips to ensure that there was no bending of the fiber during the gluing process. This would ensure that the curvatures and shape changes experienced by the LPGs were the same as the steel strips; it was also thought that the steel would help to stabilize the temperature. Using the same adhesive, these strips were fixed parallel to each other on the rubber base $75 \mathrm{~mm}$ apart. Two additional steel strips $80 \mathrm{~mm}$ long provided a perpendicular link between the sensors at either end. This framework lent longitudinal rigidity to the system, the steel having an elastic modulus of $210 \mathrm{GPa},{ }^{15}$ and this was designed to prevent the fiber (elastic modulus $\sim 70 \mathrm{GPa}$ ) from experiencing significant axial strain. The whole setup was then sealed on the sensor side of the rubber base with a room temperature vulcanizing clear silicone rubber to a depth of approximately $3 \mathrm{~mm}$. The refractive index of the silicone encapsulation was known to be greater than 1.5 as a result of previous experiments involving embedded LPGs written in standard fiber, where the attenuation bands were observed to disappear.

The PTL fiber with the LPG was fusion spliced at both ends to a standard single-mode telecom optical fiber. The spectral characteristics of the attenuation bands before and after embedding were obtained by illuminating the LPG using a broadband light source and observing the transmission spectrum with an optical spectrum analyzer (OSA) with an accuracy of $\pm 0.04 \mathrm{~nm}$ (see Fig. 2).

The two transmission spectra in Fig. 2 show that the central wavelengths of the observed attenuation bands $(1,2)$, $(1,3)$, and $(1,4)$ of the LPG have not visibly had their locations changed as a result of being embedded. The spectral profiles have altered, but not significantly. Changing the shape or position of the standard single-mode telecom optical fiber input lead alters the spectral profile (although not the position) of the LPG's attenuation bands. This effect may be due to the setting up of an interferometric cavity formed as a result of Fresnel reflections from the splices connecting the standard and PTL fibers. Further refinement of the fusion splicing of these two fibers should reduce this effect; alternatively, the PTL fiber could be used for the whole of the sensing fiber.

\section{Spectral Sensitivity of an LPG Sensor}

The LPG sensor was subjected to three measurandstemperature, compression, and bending - the sensitivities to

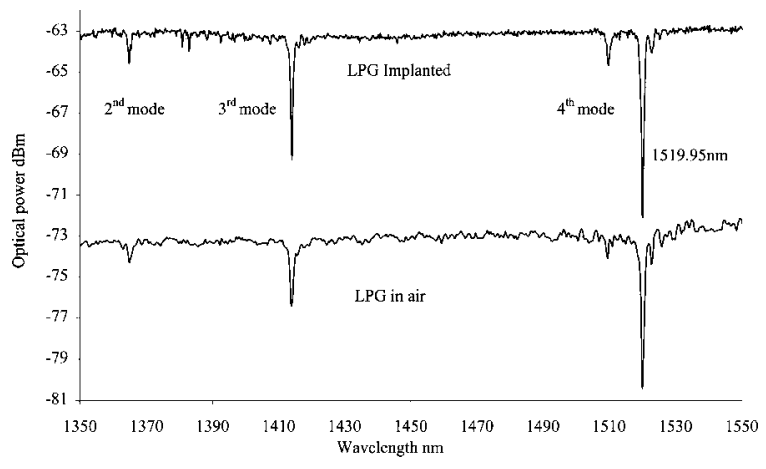

Fig. 2 The transmission spectrum of an LPG (period of $350 \times 10^{-6} \mathrm{~m}$ ) written in the PTL fiber before and after embedding; an offset of 6 $\mathrm{dBm}$ is included on the transmission spectrum of the LPG in air for clarity. 


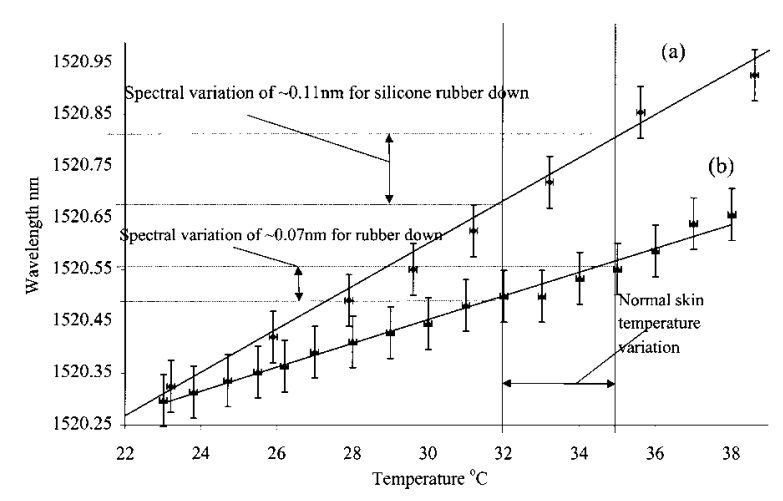

Fig. 3 The spectral sensitivity of an LPG (period of $350 \times 10^{-6} \mathrm{~m}$ ) written in the PTL fiber and embedded in the sensor. (a) The sensor's silicone rubber in contact with a hot plate. (b) The sensor's natural rubber in contact with a hot plate. The error bars indicate the accuracy of the OSA $( \pm 0.04 \mathrm{~nm})$.

all of which are important for the intended application. The sensitivity of the bare-fiber LPG to temperature had already been investigated ${ }^{14}$ by placing the fiber on a hot plate monitored using a probe with an accuracy of $\pm 0.1^{\circ} \mathrm{C}$. The spectral location of the central wavelength of the attenuation band associated with the fourth cladding mode was monitored and its sensitivity determined to be $0.04 \pm 0.005 \mathrm{~nm}{ }^{\circ} \mathrm{C}^{-1}$.

The temperature sensitivity of the sensor unit was measured by immersing the sensor in a bath of hot water and monitoring the wavelength as the water cooled. This approach gave a sensitivity of $(d \lambda / d T)=0.0952 \pm 0.5 \times 10^{-3}$ $\mathrm{nm}{ }^{\circ} \mathrm{C}^{-1}$, roughly twice that of the bare-fiber LPG. This increase in sensitivity (which for our application is of course undesirable) is due to the bonding of the fiber onto the sensor, for although the variation of refractive index with temperature will not be affected by the embedding, the axial thermal expansion of the LPG will be closer to that of the steel (typical value: $\left.5 \times 10^{-6}{ }^{\circ} \mathrm{C}^{-1}\right),{ }^{16}$ than glass (typical value: $4 \times 10^{-7}$ $\left.{ }^{\circ} \mathrm{C}^{-1}\right) .{ }^{14}$

It should be noted, however, that this way of determining the temperature sensitivity may not necessarily correspond all that well to the way in which the sensor will be used in practice. The sensing fiber is embedded inside an insulating material and in the intended application, one side of this material may well be close to body temperature while the other side is exposed to the vagaries of the environment. To illustrate the effect of this, we measured the temperature sensitivity of the sensor lying on a hot plate (as was done with the bare fiber) with the other surface exposed to room temperature. In this configuration, the temperature of the fiber will be somewhere between that of the hot plate and that of the ambient air. Measured in this way the spectral temperature sensitivity was only $0.02 \mathrm{~nm}{ }^{\circ} \mathrm{C}^{-1}$ with the natural rubber side on the hot plate and $0.04 \mathrm{~nm}{ }^{\circ} \mathrm{C}^{-1}$ with the sensor inverted. The results are illustrated in Fig. 3, along with the normal temperature variation of the skin. ${ }^{17}$ The sensor is intended to be worn close to the skin and be insulated from the surrounding air, and so this is the temperature range that the sensor must normally accommodate.

The second investigation was to determine the influence of transverse compression on the sensor. This was achieved by placing flat weights on the top surface (the silicone encapsu-

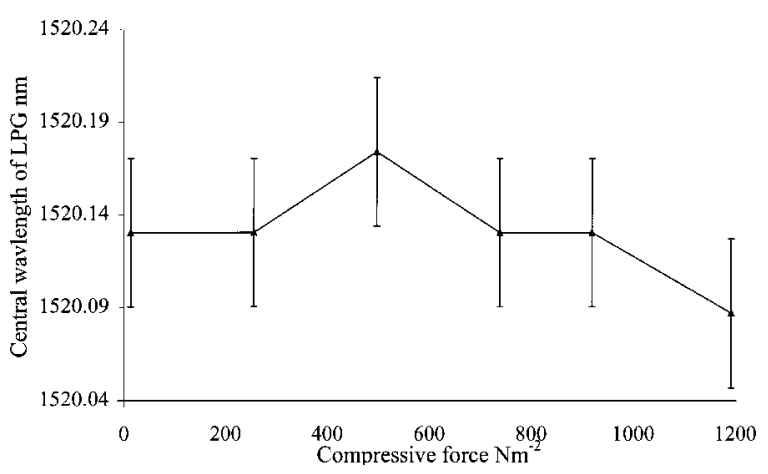

Fig. 4 Spectral stability of embedded LPG (period $350 \times 10^{-6} \mathrm{~m}$ ) under compression.

lation layer) of the sensor, which was itself placed on an optical bench (see Fig. 4). For the loads applied (up to $3.6 \mathrm{~kg}$, much higher than any load likely in the intended application), there was no observable wavelength shift or change in the spectral profile of the LPG's attenuation band. The error bars shown in Fig. 4 again indicate the accuracy of the OSA used.

The third investigation was to obtain the spectral sensitivity of the sensor to bending, and this was achieved using the experimental setup shown in Fig. 5. The sensor platform was clamped between two towers; one of the clamps was mounted on a translation stage, which was moved inward to induce a bend in the optical fiber. For this arrangement where the LPG is midway between the clamps, the sensor's curvature, $R$, is given by ${ }^{18}$

$$
R=\frac{2 \times d}{\left(d^{2}+L^{2}\right)},
$$

where $L$ is the half-distance between the edges of the two towers and $d$ is the bending displacement at the center of the LPG.

The results for this experiment, along with a theoretically predicted wavelength shift, are shown in Fig. 6. The theoretical wavelength shift was obtained using the same approach as in Ref. 19 and is given by

$$
\Delta \lambda=\left[\frac{\lambda}{\left(\delta n_{\mathrm{eff}}-\delta n_{g}\right)} \times \frac{\mathrm{d} \delta n_{\mathrm{eff}}}{\mathrm{d} R}+\frac{\left(\delta n_{\mathrm{eff}}\right)^{3}}{\delta n_{g}} \frac{\mathrm{d} \Lambda}{\mathrm{d} R}\right] \Delta R,
$$

where $\delta n_{\text {eff }}=n_{\text {coeff }}-n_{\text {cleff } \nu}$ is the differential effective index between the cladding and the core mode and $\delta n_{g}=n_{\operatorname{cog}}$ $-n_{\text {clg } \nu}$ is the differential group index. The effective refractive indices of the core and fourth cladding mode were calculated as a function of curvature using a 2-D curvilinear hybrid mode eigenvalue equation ${ }^{20,21}$ using the optical constants

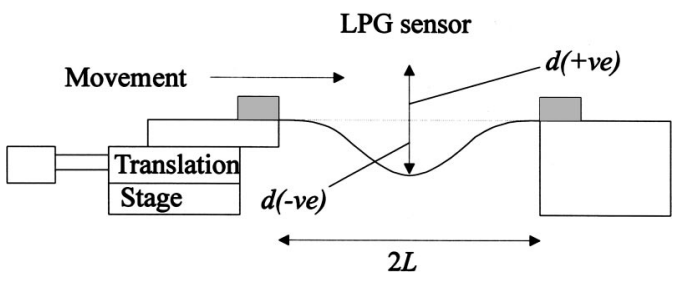

Fig. 5 Schematic of bending apparatus. 


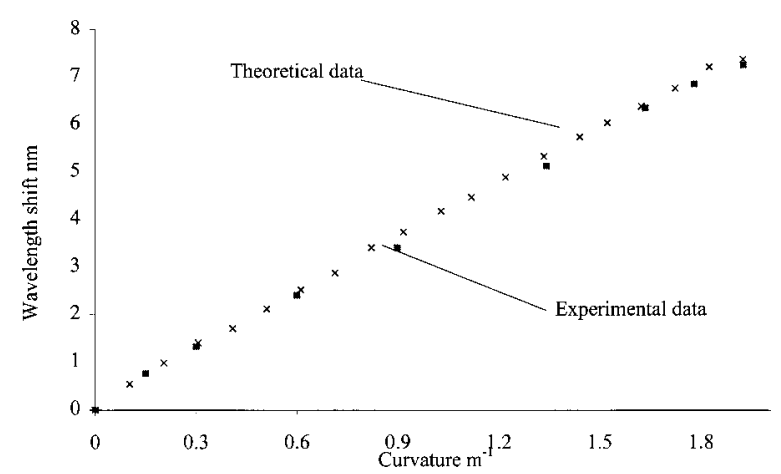

Fig. 6 The central wavelength of the attenuation band (fourth mode) of the LPG with period $350 \times 10^{-6} \mathrm{~m}$ embedded in the sensor as a function of curvature.

given in Ref. 14 and assuming that the amount of birefringence induced in a typical single-mode fiber is negligibly small for curvatures of $<2 \mathrm{~m}^{-1}$ (no splitting of the attenuation bend). Figure 6 demonstrates reasonable agreement between the theoretical wavelength shift calculated at a number of different curvatures and the experimental results. Note that the small amount of "noise" visible on the theoretical data is a result of computational truncation errors, owing to the limited precision of the Mathcad software. Mathcad performs all computations in double precision (floating point: 64 bits), which causes errors to occur when using the built-in Bessel functions and their cross products with small arguments. Over the range of curvature investigated, the spectral sensitivity of the sensor was found to be linear, with $(\mathrm{d} \lambda / \mathrm{d} R)=3.747$ $\pm 0.006 \mathrm{~nm} \mathrm{~m}$.

\section{Respiratory Monitoring}

In order to validate this sensor design for the intended application, a commercial manikin used as a resuscitation training aid was employed. ${ }^{22}$ This manikin consists of a rigid underframe over which is stretched a polymer skin. An air bag is placed between the frame and skin and when inflated or deflated is used to simulate expansion and contraction of the surface of the torso in volumetric proportions similar to those of breathing. This provides a test platform for changes in dimension and shape similar to those that are expected in humans.

The response of the sensor was recorded at various states of inflation at five different orientations on the manikin. Each location is represented by a set of dimensions as indicated in Fig. 7 and Table 1. The shift in central wavelength was also recorded with reference to the peripheral expansion of the dummy's skin in Fig. 8 and Fig. 9. The variation in response apparent between the upper and lower chest regions mimics that which might be expected in a real subject since the expansion of the rib cage has a more significant contribution at higher levels of ventilation. ${ }^{23}$ In our experiment, however, this phenomenon can be attributed to the profile and position of the internal airbag.

An inspection of Fig. 8 and Fig. 9 shows that the spectral response of the LPG sensor as a function of peripheral expansion of the manikin's skin varies from position to position. The errors illustrated in these figures correspond to the spec-

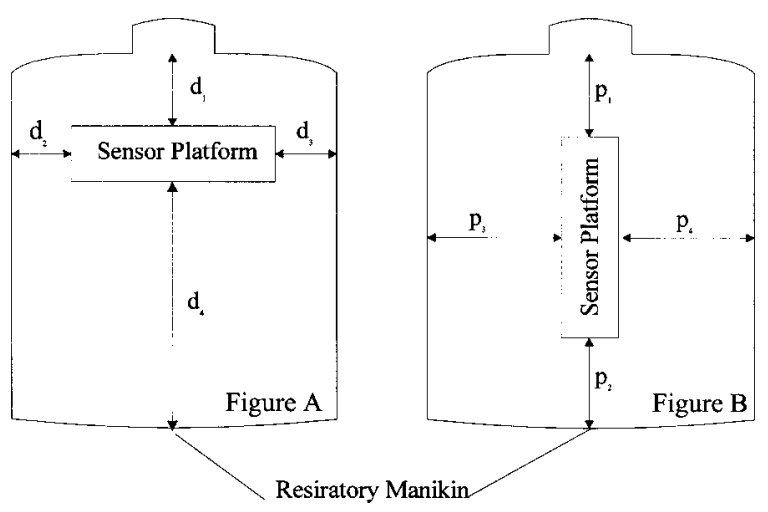

Fig. 7 The positioning of the LPG sensor platform on the manikin.

tral accuracy of the OSA $( \pm 0.04 \mathrm{~nm})$ and a circumference error of $\pm 1 \mathrm{~mm}$. The circumference error is an estimate of the variation of the manikin's skin deformation between each set of results. The curves shown in Fig. 8 and Fig. 9 are presented simply as guides for the eye to better illustrate the different responses at the various locations.

As noted earlier, the sensor is temperature sensitive. In normal use it would be worn next to the skin and insulated from the environment, so that it would be subjected to a likely temperature variation of only 32 to $35^{\circ} \mathrm{C} .{ }^{17}$ The maximum wavelength uncertainty associated with this temperature range is about $\pm 0.14 \mathrm{~nm}$ (using the temperature sensitivity of 0.095 $\mathrm{nm}{ }^{\circ} \mathrm{C}^{-1}$ ). Combining this with the curvature resolution of $\pm 1.1 \times 10^{-2} \mathrm{~m}^{-1}$ for the sensing scheme leads to a relative error in the circumference that varies between \pm 0.3 and $\pm 2.3 \%$, corresponding, respectively, to positions 1 and 3 on the manikin.

To improve on the accuracy, we plan to investigate two approaches to dealing with the temperature cross-sensitivity. First the steel supports are to be replaced by carbon-fiber sheets in which the thermal expansion coefficient is typically in the range of $\pm 4 \times 10^{-7}{ }^{\circ} \mathrm{C}^{-1}$, depending on the material's composition. This should in principle allow the temperature sensitivity of the sensor platform to be significantly reduced. Second, a fiber Bragg grating may be used to discriminate between bending and temperature. ${ }^{24}$

A conservative estimate of the number of sensors required for the intended application was made using chest profile data obtained from a computed tomography (CT) imager. A singleline profile from the upper chest of a male subject was chosen because it constituted the most complex geometry from the image data. This profile was then modeled using a seventhorder polynomial, and the profile length of the curve was evaluated symbolically as a line integral to give the crosssectional area and then squared for the volumetric error. This value was then compared with the same line evaluated numerically (simple trapezoidal integration) at varying discrete intervals along the profile. Assuming that an identical longitudinal and lateral resolution is needed to represent the overall profile of the torso by squaring the results, Fig. 10 depicts the total number of sensors required versus volumetric error. This error may be reduced further with the application of cubicspline interpolation. Linking this to generally accepted volumetric performance standards for spirometric devices, ${ }^{25}$ about 
Table 1 The measured central wavelength shift of the LPG sensor at various positions on the manikin.

\begin{tabular}{lccccc}
\hline $\begin{array}{l}\text { Position on Dummy } \\
\text { Fig. 7(a) }\end{array}$ & $\begin{array}{c}d_{1} \\
(\mathrm{~mm})\end{array}$ & $\begin{array}{c}d_{2} \\
(\mathrm{~mm})\end{array}$ & $\begin{array}{c}d_{3} \\
(\mathrm{~mm})\end{array}$ & $\begin{array}{c}d_{4} \\
(\mathrm{~mm})\end{array}$ & $\begin{array}{c}\text { Maximum detected wavelength shift } \\
(\mathrm{nm})\end{array}$ \\
\hline 1 & 90 & 50 & 50 & 250 & 1.05 \\
2 & 185 & 50 & 50 & 185 & 3.18 \\
3 & 287 & 50 & 50 & 75 & 0.95 \\
$\begin{array}{l}\text { Position on Dummy } \\
\text { Fig. 7(b) }\end{array}$ & $\begin{array}{l}p_{1} \\
(\mathrm{~mm})\end{array}$ & $\begin{array}{l}p_{2} \\
(\mathrm{~mm})\end{array}$ & $\begin{array}{l}p_{3} \\
(\mathrm{~mm})\end{array}$ & $\begin{array}{l}p_{4} \\
(\mathrm{~mm})\end{array}$ & $\begin{array}{c}\text { Maximum detected wavelength shift } \\
(\mathrm{nm})\end{array}$ \\
\hline 4 & 100 & 25 & 165 & 165 & 1.98 \\
5 & 205 & 128 & 165 & 165 & 1.70 \\
\hline
\end{tabular}

twenty such sensors would be required for this application using cubic-spline interpolation. Of course the resuscitation manikin provides only a simple approximation to the behavior of the human torso during respiration. The location and number of sensors required to monitor respiratory function within acceptable standards ${ }^{25}$ will need to be investigated experimentally using volunteers instrumented with an array of improved sensor platforms working in conjunction with a suitable multiplexing and interrogation scheme.

There are several possible multiplexing and interrogation techniques that could be used. We have considered the use of a broadband light source illuminating a series of in-line LPGs combined with a miniature fiber-optic spectrometer (e.g., those from Ocean Optics ${ }^{26}$ ) to record the transmission spectra of the LPGs. Our concern is that the limited spectral resolution of these spectrometers would seriously limit the curvature resolution of the interrogation scheme. The sensing scheme that is being investigated is based upon a derivative spectroscopy technique in which an in-line series of sensing LPGs are being interrogated by spectrally matched fiber Bragg gratings. ${ }^{27}$

For the intended application, a number of alternative (nonoptical) sensing elements could also in principle be used. Most schemes are based on strain measurement (e.g., capacitance $^{28}$ and resistance ${ }^{29}$ gauges or bistable switches ${ }^{30}$ ) and as such are not directly applicable to surface profile reconstruction because a dynamic model of the motion of the

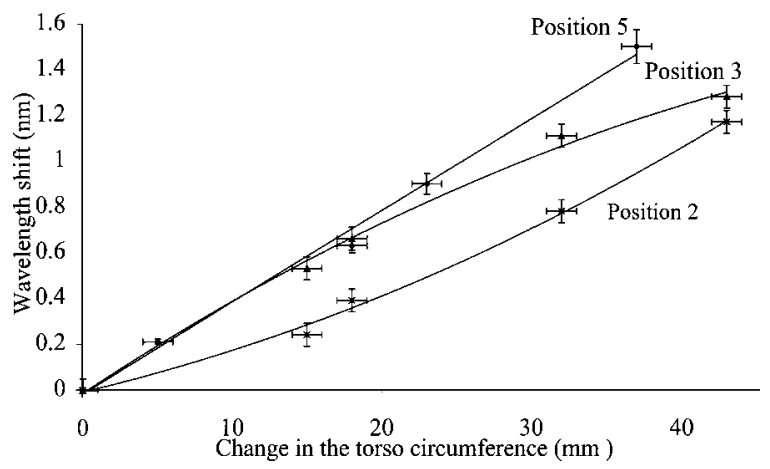

Fig. 8 Spectral functionality of the LPG sensor positioned on the lower chest. thorax and abdomen during breathing would be required on which localized strain measurement could be used to infer changes in geometry. It is a considerable task to create a model that would accurately describe respiratory interactions over a broad range of subjects. The solution detailed here sidesteps the need for such a model because geometry is the fundamental measurand and the chest surface profile can be obtained directly from sensor signals.

While other optical-based bend-sensing elements have been developed (e.g., the Shape Tape developed by the Canadian Space Agency ${ }^{31}$ ), these do not possess the ability to be serially multiplexed, relying as they do on curvaturedependent attenuation, and commercially available configurations of these sensors do not lend themselves to this application because they are arranged linearly on one strip of steel, and that platform cannot be stretched. The curvature resolution of the Shape Tape is about $10^{-3} \mathrm{~m}^{-1}$, which is comparable to the interrogation scheme we have under investigation. ${ }^{27}$ Our approach allows the simple multiplexing of the sensors, providing the ability to scale up the approach to multiple sensors without a dramatic increase in the size of the peripheral electronics.

\section{Conclusion}

The spectral characteristics of an embedded long-period grating fabricated in a PTL fiber were investigated. The spectral

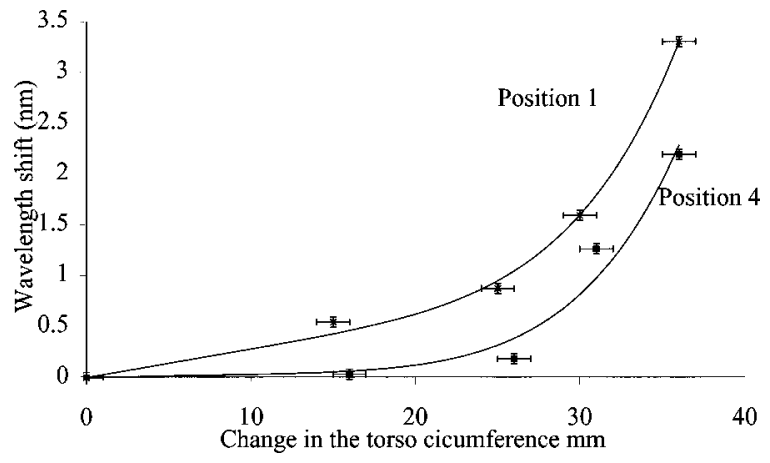

Fig. 9 Spectral functionality of the LPG sensor positioned on the upper chest. 


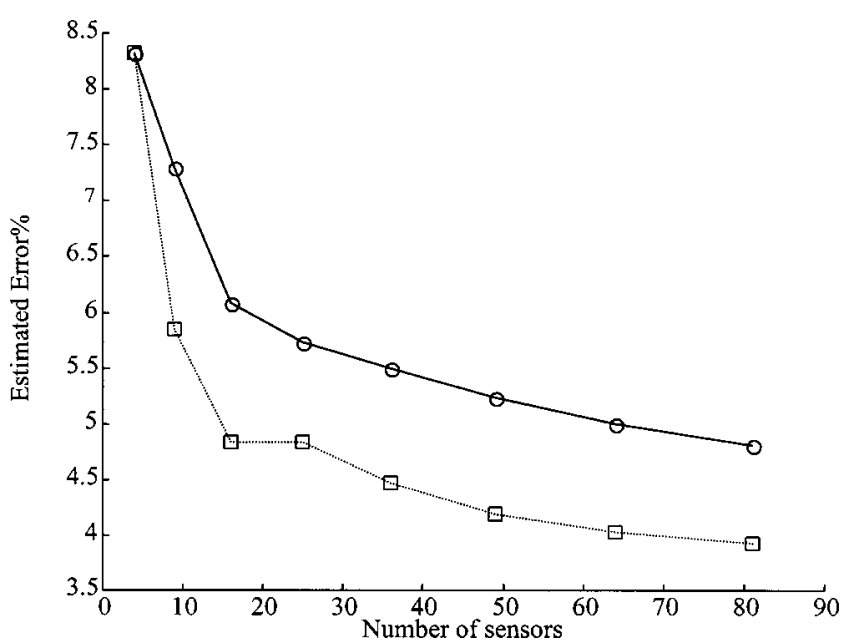

Fig. 10 Density of sensing positions versus volumetric error for reconstructed CT scan chest profile data. Solid and broken curves represent the errors from the trapezoidal approximation and 4-point cubic spline interpolation, respectively.

location and profile of the attenuation bands were found to be unaffected by embedding the LPG, although the temperature sensitivity was increased. A curvature spectral sensitivity of $3.747 \mathrm{~nm} \mathrm{~m}$ with a curvature resolution of $\pm 1.1 \times 10^{-2} \mathrm{~m}^{-1}$ was found for this sensor. Axial strain for this sensor in the intended application can be assumed to be negligible because the sensor is fixed to an axially rigid steel support.

Normal skin temperature variations limit the accuracy of the sensor to a worst-case equivalent error in a circumference measurement of $\pm 2.3 \%$. Where this is insufficient or where it cannot be guaranteed that the sensor will be in contact with the skin, we have identified two avenues toward improving or controlling the temperature sensitivity-the use of a carbon fiber support frame or the incorporation of an additional Bragg grating temperature sensor.

It has also been demonstrated that such a sensor can be used to distinguish among the geometric variations associated with different locations on a simulated torso during respiratory movement. We have shown that, in theory, an array of approximately twenty such sensors should be sufficient to generate a geometric profile of the chest and abdomen in three dimensions with the necessary accuracy.

\section{Acknowledgment}

The authors would like to thank Tim Hart of Fibercore Ltd. for fruitful discussions and supplying the material constants and specification of the PTL fiber. We also thank M. R. Miller M.D., Consultant in respiratory medicine, Selly Oak Hospital, Birmingham, UK, for the computed tomography image data. This work was funded by the UK Engineering and Physical Sciences Research Council.

\section{References}

1. S. Vasilev and O. Medvedkov, "Long-period refractive index fiber gratings: properties, applications and fabrication techniques," Proc. SPIE 4083, 212-223 (2000).

2. Y. Ruan, X. Huang, Y. Jiang, and J. Wang, "The design and simulation of cascade long-period fiber gratings for flattening EDFA's gain," Proc. SPIE 3801, 240-245 (1999).

3. A. Vengsarkar, P. Lemaire, J. Judkins, V. Bhatia, and T. Erdogan,
"Long-period gratings as band-rejection filters," J. Lightwave Technol. 14(1), 58-64 (1996).

4. V. Bhatia and A. M. Vengsarkar, "Optical fibre long-period grating sensors," Opt. Lett. 21(9), 692-694 (1996).

5. H. J. Patrick, A. D. Kersey, and F. Bucholtz, "Analysis of the response of long period fiber gratings to external index of refraction," J. Lightwave Technol. 16(9), 1606-1612 (1998).

6. V. Grubsky and J. Feinberg, "Long-period gratings with variable coupling for real-time sensing applications," Opt. Lett. 25(4), 203-205 (2000).

7. V. Bhatia, T. D'Alberto, N. Zabaronick, and R. Claus, “Temperatureinsensitive and strain-insensitive long-period gratings sensor for smart structures," Opt. Eng. 36, 1872 (1997).

8. R. P. Espindola, R. Windeler, A. Abramov, B. Eggleton, T. Strasser, and D. DiGiovanni, "External refractive index insensitive air-clad long period fibre grating," Electron. Lett. 35(4), 327-328 (1999).

9. L. Dong, L. Reekie, and J. L. Cruz, "Long period gratings formed in depressed cladding fibers," Electron. Lett. 33(22), 1897-1898 (1997).

10. J. Jang, Se Yoon Kim, Sun-Wook Kim, and Min-Sung Kim, "Temperature insensitive long-period fibre gratings," Electron. Lett. 35(24), 2134-2136 (1999).

11. T. A. Wilson, K. Rehder, S. Krayer, E. Hoffman, C. Whitney, and J. Rodarte, "Geometry and displacement of human rib," J. Appl. Physiol. 62(5), 1872-1877 (1987).

12. T. Earthrowl-Gould and B. Jones "Chest and abdominal surface motion measurement for continuous monitoring of respiratory function," in Proceedings of ImechE, Part H, Engineering in Medicine (2001).

13. Y. Liu, L. Zhang, and I. Bennion, "Design and realization of longperiod grating devices in conventional and high birefringence fibers and their novel applications as fiber-optic load sensors," IEEE J. Sel. Top. Quantum Electron. 5(5), 1373-1378 (1999).

14. T. Allsop, D. J. Webb, and I. Bennion, "Investigations of the spectral sensitivity of long period gratings fabricated in 3-layered optical fiber," J. Lightwave Technol. 21(1), 264-268 (2003).

15. A. Wolfenden, G. M. Ludtka, and L. S. Cook, "An evaluation of three methods to measure the dynamic elastic modulus of steel," $J$. Test. Eval. 23(3), 176-179 (1995).

16. R. D. Harrison, Book of Data: Chemistry, Physical, Science Physics. Penguin, Bristol, UK (1978).

17. T. Miyanaga, W. Urabe, and Y. Nakano, "Simplified human bodymodel for evaluating thermal radiant environment in a radiant cooled space," Build Env. 36(7), 801-808 (2001).

18. W. Du, H. Tam, M. Liu, and X. Tao, "Long-period fiber grating bending sensors in laminated composite structures," in Sensory Phenomena and Measurement Instrumentation for Smart Structures and Materials, Proc. SPIE 3330, 284-292 (1998)

19. V. Bhatia, "Applications of long-period gratings to single and multiparameter sensing," Opt. Express 4(11), 457-466 (1999).

20. C. Tsao, Optical Fibre Waveguide Analysis, Oxford, New York (1992).

21. J. Ma, W. Tang, and W. Zhou, "Optical-fibre sensor for simultaneous measurement of pressure and temperature: analysis of cross sensitivity," Appl. Opt. 35(25), 5206-5210 (1996).

22. Little Anne CPR Manikin, Laerdal Medical, http://www.laerdal.co.uk

23. K. Konno and J. Mead, "Measurement of the separate volume changes of the rib cage and abdomen during breathing," J. Appl. Physiol. 22(3), 407-422 (1967).

24. H. Patrick G. Williams, A. Kersey, J. Pedrazzani, and A. Vengsarkar, "Hybrid fiber Bragg grating/long period fiber grating sensor for strain/temperature discrimination," Photon. Technol. Lett. 8(9), 1223-1225 (1998).

25. American Thoracic Society, ATS Statement-Snowbird Workshop on Standardization of Spirometry, Am. Rev. Respir. Dis. 119, 831-838 (1979).

26. USB2000 Fiber Optic Spectrometer, Ocean Optics, Inc., http:// www.oceanoptics.com

27. T. Allsop, T. Earthrowl-Gould, R. Reeves, D. J. Webb, and I. Bennion, "The interrogation/multiplexing of long period grating curvature sensors using derivative spectroscopy technique based upon fiber Bragg gratings" (in preparation).

28. R. P. Kampen, "Bulk-micromachined capacitive servoaccelerometer," Ph.D. Thesis, Laboratory of Electronic Instrumenta- 
Allsop et al.

tion, Delft Univer-sity of Technology, Delft University Press, Delft, The Netherlands (1995).

29. Strain gauges, Minebea Co. Ltd., http://www.minebea.co.jp

30. U.S. Patent \#5563458, Apparatus and method for sensing surface flexure sensing shape and motion with bistable switches that digitize material strain caused by flexure.

31. Shape Tape, Measurand Shape Advantage, http:// 